\title{
The Search for Neutrinoless Double Beta Decay with CUORE
}

\section{Jonathan Ouellet ${ }^{*}$}

Massachusetts Institute of Technology

E-mail: ouelletj@mit.edu

CUORE is a $741 \mathrm{~kg}$ bolometric array of $988 \mathrm{TeO}_{2}$ crystals designed to search for the neutrinoless double beta decay of ${ }^{130} \mathrm{Te}$. The detector is in the final stages of construction at the Laboratori Nazionali del Gran Sasso in Italy, where it will start taking data in 2016. With a target background of 0.01 counts/( $\mathrm{keV} \cdot \mathrm{kg} \cdot \mathrm{y})$, we expect that in five years of data taking CUORE will have a sensitivity to a ${ }^{130} \mathrm{Te} 0 v \beta \beta$ half-life of $9.6 \times 10^{25} \mathrm{y}$. The first phase of CUORE, called CUORE- 0 finished taking data in March 2015, and its performance gives confidence that CUORE is on track to achieve its goals. Here, we discuss the final results of CUORE-0, their implications for CUORE, and the current status of the CUORE experiment.

XIII International Conference on Heavy Quarks and Leptons

22-27 May, 2016

Blacksburg, Virginia, USA

* Speaker.

${ }^{\dagger}$ On behalf of the CUORE collaboration. 


\section{Introduction}

It has been more than a decade since the first neutrino oscillation experiments proved that neutrinos are massive particles $[1,2,3,4,5,6]$. However, we still do not know conclusively, how to incorporate the neutrino mass into the Standard Model (SM). The neutrino could get its mass through the same Higgs mechanism which gives mass to all the other fermions in the SM - a Dirac type mass - or the neutrino could be unique among the fermions, getting its mass through a different mechanism entirely - a Majorana type mass. This latter possibility would have deep implications for our understanding of nature, from providing a window into new physics at higher energy scales to implications about the formation of the matter asymmetry of the Universe fractions of a second after the Big Bang.

Neutrinoless Double-Beta $(0 v \beta \beta)$ Decay, $(Z, A) \rightarrow(Z+2, A)+2 \beta$, is an as-yet undiscovered process that could provide an answer to the question of the Majorana nature of the neutrino. If discovered, it would indicate conclusively that Lepton number is not a conserved quantity of nature, that the neutrino is a Majorana fermion — and thus is its own anti-particle — and provide insight into both the neutrino mass hierarchy and absolute mass scale. In recent years, $0 v \beta \beta$ decay has garnered a lot of experimental interest, with the largest experiments focusing on the isotopes ${ }^{76} \mathrm{Ge}$ [7], ${ }^{136} \mathrm{Xe}[8,9]$, and ${ }^{130} \mathrm{Te}[10,11]$.

The $0 v \beta \beta$ decay rate of an isotope can be parameterized as

$$
\Gamma^{0 v}=\ln 2 G_{0 v}\left(Q_{\beta \beta}\right)\left|\mathscr{M}_{0 v}\right|^{2}\left|f_{0 v}\right|^{2} .
$$

Where $G^{0 v}\left(Q_{\beta \beta}\right)$ is a precisely calculable phase space factor, which depends on the fifth power of the total energy of the decay, $Q_{\beta \beta}$. The $\mathscr{M}_{0} v$ term is the nuclear matrix element (NME), and at present, has a relatively large uncertainty. All of the beyond the SM physics is encoded by $\left|f_{0 v}\right|^{2}$. In the simplest models, this term is interpreted as depending on the effective Majorana mass, $m_{\beta \beta}$,

$$
\left|f_{0 v}\right|=\frac{m_{\beta \beta}}{m_{e}}=\frac{1}{m_{e}}\left|\sum_{i} U_{e i}^{2} m_{i}\right| .
$$

Where $U_{e i}$ are the elements of the PMNS matrix, and $m_{i}$ are the neutrino masses. The allowed parameter space for $m_{\beta \beta}$ depends on the mass hierarchy, and the two allowed regions are shown in Fig. 4. The goal of the CUORE experiment, is to probe $m_{\beta \beta}$ down to the top of the region allowed in the inverted hierarchy, $m_{\beta \beta} \sim 50 \mathrm{meV}$.

The CUORE experiment is a ton-scale search for the $0 v \beta \beta$ decay of the isotope ${ }^{130} \mathrm{Te}$. The predecessor to CUORE, called Cuoricino, ran from 2003-2008 in Hall A of the Laboratori Nazionali del Gran Sasso (LNGS) in Italy. Until recently, Cuoricino held the best limit on the ${ }^{130} \mathrm{Te} 0 v \beta \beta$ decay half-life at $2.8 \times 10^{24} \mathrm{yr}\left(90 \%\right.$ C.L.), based on a $19.75 \mathrm{~kg} \cdot \mathrm{yr}^{130} \mathrm{Te}$ exposure [12].

In this proceeding, we discuss the results of CUORE-0, which completed in 2015 and the implications that can be learned for CUORE. We also discuss the current status of the CUORE detector construction.

\section{The CUORE Experiment}

\subsection{A CUORE Bolometer}

The CUORE detector is an array of 988 bolometers each operated independently as an indi- 

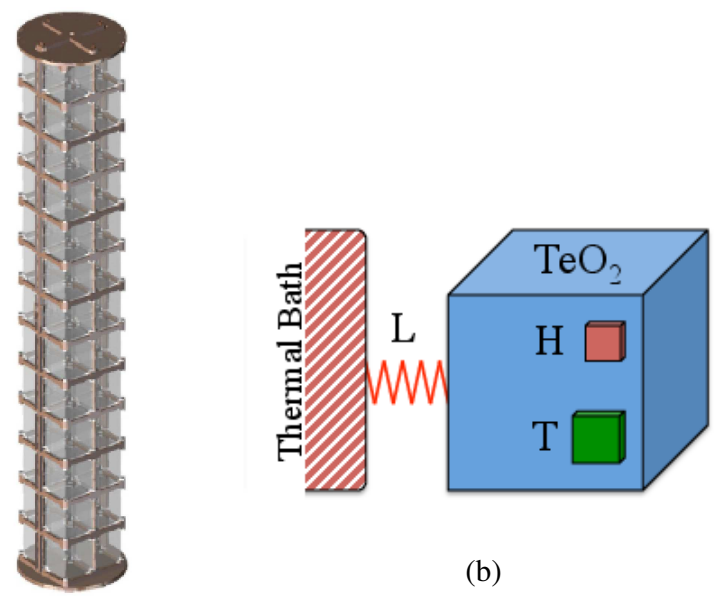

(b)

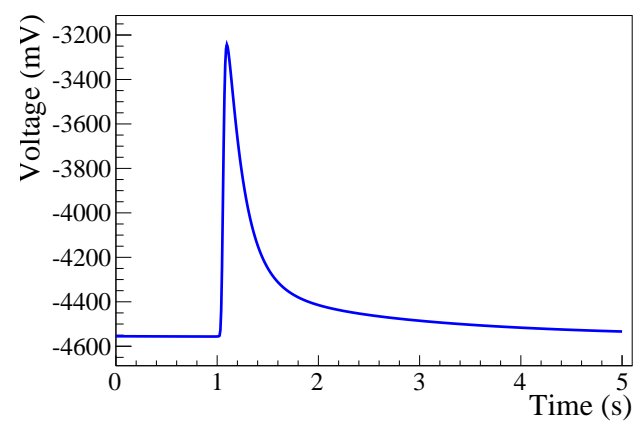

(c)

(a)

Figure 1: (a) CUORE-0 tower array rendering. The tower consists of 13 floors of 4 bolometers, mounted in a copper frame. (b) Schematic of a single CUORE-0 bolometer showing the thermistor $(\mathrm{T})$, the heater $(\mathrm{H})$, and the weak thermal link (L) between $\mathrm{TeO}_{2}$ crystal and copper thermal bath (not to scale). (c) An example of a bolometer signal with an energy of approximately $2615 \mathrm{keV}$. The rise and fall times of this signal are $0.05 \mathrm{~s}$ and $0.2 \mathrm{~s}$ respectively. Figure from [13].

vidual search for $0 v \beta \beta$ decay. A CUORE bolometer is composed of two main components, the absorber which absorbs the energy released in a particle interaction and converts that energy deposition into a change in temperature and the thermistor which converts this change in temperature into a measurable change in voltage.

A CUORE absorber is a $5 \times 5 \times 5 \mathrm{~cm}^{3} \mathrm{TeO}_{2}$ crystal weighing approximately $750 \mathrm{~g}$. The crystal is made from ${ }^{\text {nat }} \mathrm{Te}$, which is $\sim 34 \%{ }^{130} \mathrm{Te}$, and thus acts as both the source and detector of the decays of interest. The crystals are arranged into an array of 19 towers, 13 floors each, with 4 crystals per floor. The CUORE detector will have a total active mass of $741 \mathrm{~kg}$, for $206 \mathrm{~kg}$ of ${ }^{130} \mathrm{Te}$. The copper support frames act as the bolometers' fixed temperature heat bath. The detector is held at an operating temperature of $\sim 10 \mathrm{mK}$ in a dilution refrigerator. At this low temperature, the heat capacity of a single crystal is such that a $1 \mathrm{MeV}$ energy deposition corresponds to a change in temperature of about $100 \mu \mathrm{K}$. This small change in temperature is read out using a Neutron Transmutation Doped Germanium thermistor (NTD). These thermistors have a resistivity which is exponentially dependent on temperature, and amplify the $\sim 1 \%$ temperature change to a change in resistivity of $\sim 10 \%$. The NTDs have typical resistances of $\sim 100 \mathrm{M} \Omega$ and are current biased and read out using room temperature electronics.

\subsection{Background Mitigation Strategy}

One of the primary goals in the design and assembly of CUORE was to decrease the background rate in the region of interest (ROI) from that seen in Cuoricino. For CUORE, we aimed for a maximum background rate of 0.01 counts/(keV·kg $\cdot \mathrm{yr})$ at the full energy of the ${ }^{130} \mathrm{Te}$ decay, $Q_{\beta \beta}=2527 \mathrm{keV}$. We worked closely with the $\mathrm{TeO}_{2}$ crystal grower at the Shanghai Institute of Ceramics, Chinese Academy of Science to limit both the surface and bulk contamination of the crys- 
tals themselves [14]. We measured the bulk and surface contamination to be less than $5.0 \times 10^{-7}$ and $1.3 \times 10^{-6} \mathrm{~Bq}$ per crystal, respectively, in ${ }^{238} \mathrm{U}$ and $6.3 \times 10^{-7}$ and $3.0 \times 10^{-7} \mathrm{~Bq}$ per crystal, respectively, in ${ }^{232} \mathrm{Th}$ (90\% C.L.) [15]. We transported the crystals from Shanghai to LNGS at sea level in order to minimize cosmogenic activation.

To reduce the amount of contamination from the supporting structure of our detector, we developed and tested strict material selection and cleaning procedures. By redesigning the tower support structure, we reduced the amount of copper needed per crystal by a factor of about $\sim 3$. To remove the contamination on the surface of the copper facing our detectors, we developed a copper treatment procedure that combines tumbling, electropolishing, chemical etching and magnetic plasma etching. The result of this treatment reduced the total surface contamination of the copper to below $1.3 \times 10^{-7} \mathrm{~Bq} / \mathrm{cm}^{2}$ at $90 \%$ C.L. in both ${ }^{238} \mathrm{U}$ and ${ }^{232} \mathrm{Th}$ [16]. To minimize re-contamination from radon in the air, we assemble and store all of the towers in glove boxes under constant $\mathrm{N}_{2}$ flux in a class 1000 clean room.

\section{Results of the CUORE-0 Experiment}

In addition to the 19 towers produced for CUORE, a $20^{\text {th }}$ tower was produced - the first tower off the assembly line - and was operated in the old Cuoricino cryostat as an independent experiment called CUORE-0. It was originally foreseen as a full scale debugging of the assembly procedure for CUORE, but is a formidable $0 v \beta \beta$ decay search in its own right. A full description of the detector construction and commissioning can be found in [17].

CUORE-0 collected $0 v \beta \beta$ search data from March 2013 to March 2015 for a total exposure of $35.2 \mathrm{~kg} \cdot \mathrm{yr}$ or $9.8 \mathrm{~kg} \cdot \mathrm{yr}$ of ${ }^{130} \mathrm{Te}$ exposure. In March 2015 , with less than half the run time, the expected sensitivity of CUORE-0 had surpassed the limit set by Cuoricino and CUORE-0 data collection stopped.

\subsection{Detector Performance}
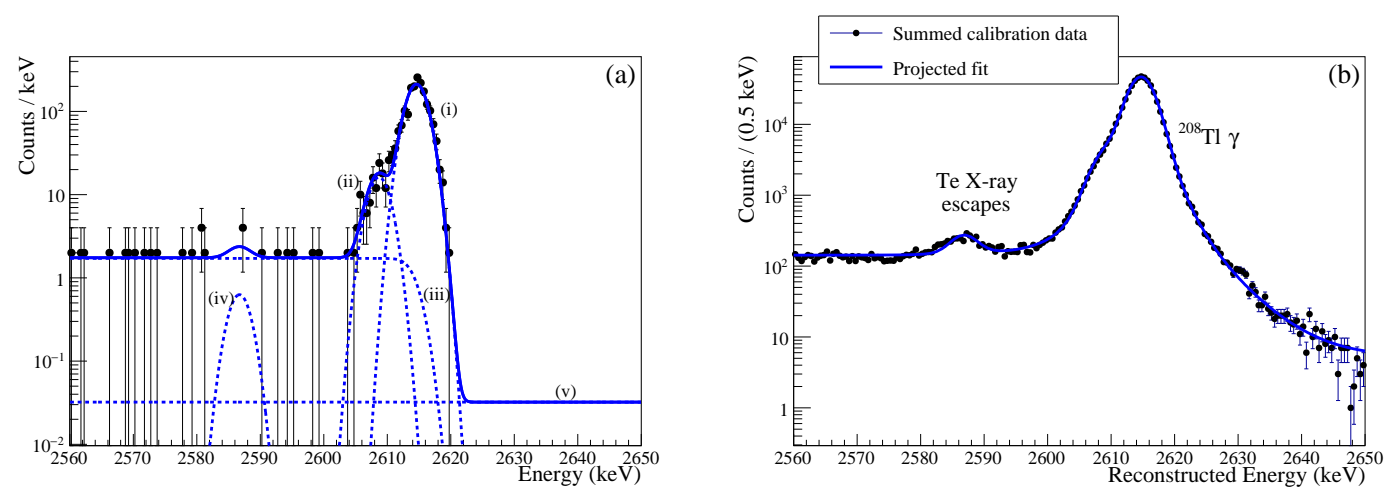

Figure 2: Fit to the ${ }^{208} \mathrm{Tl}$ photopeak in the calibration data. (a) The fit to a single channel and dataset with the individual components for (i) the ${ }^{208} \mathrm{Tl}$ photopeak, (ii) the sub-peak, (iii) a compton edge (iv) the $30 \mathrm{keV}$ $\mathrm{X}$-ray escape peak and (v) a flat background. (b) The same fit summed over all channels and datasets. The FWHM of the fit is $4.9 \mathrm{keV}$ with an effective resolution over all channels and datasets of $5.1 \mathrm{keV}$ (FWHM). Figure adapted from [10]. 
The data collection for CUORE-0 is split into datasets. Each dataset is about one month long with the first and last few days devoted to calibration. The calibration data are shared between adjacent datasets. We calibrate the detectors by inserting thoriated tungsten strings inside the outermost lead shielding. We use the $\gamma$ lines from the thorium daughters to determine a calibration function for each channel. The highest $\gamma$ calibration line comes from ${ }^{208} \mathrm{Tl}$ decay at $2615 \mathrm{keV}-$ only $87 \mathrm{keV}$ above from our ROI. Since the rate of the background $\gamma$ lines in our $0 v \beta \beta$ decay search data is very low, we use this line in the calibration data to determine our energy resolution for each channel in each dataset.

We fit the ${ }^{208} \mathrm{Tl}$ calibration line with a phenomenologically determined form, which consists of a single gaussian for the photopeak, a smaller gaussian $-\sim 5 \%$ in amplitude - about $5 \mathrm{keV}$ lower in energy, an Erfc function to accomodate a compton edge, a third gaussian $\sim 30 \mathrm{keV}$ lower to account for a $30 \mathrm{keV}$ Te X-ray escape, and a flat background. (See Fig. 2). We perform a simultaneous unbinned extended maximum likelihood (UEML) fit to fit all channels and datasets simultaneously [11]. The energy resolution of the summed fit is $4.8 \mathrm{keV}$ full-width half-max (FWHM), and the physics-exposure weighted energy resolution is $4.9 \mathrm{keV}$ FWHM. We project the fitted detector response into the $0 v \beta \beta$ ROI [11] and estimate the resolution at $Q_{\beta \beta}$ to be $5.1 \pm 0.3 \mathrm{keV}$ FWHM, accounting for both energy dependence and differences between the physics data and the calibration.

\subsection{Results}
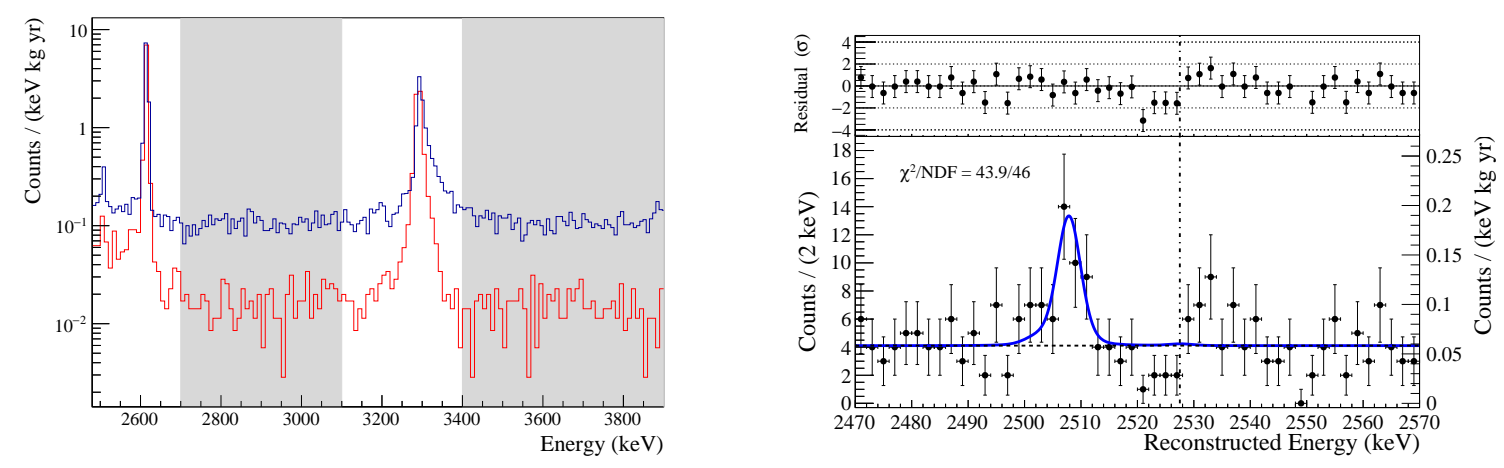

Figure 3: Left: The $\alpha$-continuum region for CUORE-0 (red line) and Cuoricino (blue line). The $\alpha$ rate is evaluated over the shaded regions from $2700-3900$ excluding the ${ }^{190} \mathrm{Pt} \alpha$-peak. Right: The best fit model (solid blue line) overlaid the CUORE-0 data (black points). The data are shown with gaussian error bars. The peak at $2507 \mathrm{keV}$ is due to ${ }^{60} \mathrm{Co}$ and the dash-dotted line indicates the expected position of a $0 v \beta \beta$ signal. The dashed black line indicates the continuum background in the ROI. The normalized residuals are shown above.

The analysis techniques are presented in [11] and the final results in [10]. We measured the $\alpha$-rate in the ROI by measuring the event rate in the region from $2700-3900 \mathrm{keV}$, excluding the ${ }^{190} \mathrm{Pt} \alpha$-line. We call this region the $\alpha$-continuum since it is nearly flat, dominated by degraded $\alpha$ decays, and can be extrapolated down into the ROI. We measured the rate in this region to be $b_{\alpha}=$ $0.016 \pm 0.001 \mathrm{counts} /(\mathrm{keV} \cdot \mathrm{kg} \cdot \mathrm{yr})$. This is a factor of 6.8 improvement over the background seen in 
Cuoricino of $0.110 \pm 0.001$ counts $/(\mathrm{keV} \cdot \mathrm{kg} \cdot \mathrm{yr})$. This is consistent with the expected improvement due to the background mitigation procedures.

The background in the ROI, has two components: the $\alpha$ component, which we measure in $\alpha$-continuum, and a component from multi-scattered $\gamma$-decays. The $\gamma$ component originates in the materials of the cryostat, and so is not expected to change significantly from Cuoricino, and indeed the measured rate in CUORE-0 is consistent with that seen in Cuoricino. This background is, however, expected to decrease significantly in CUORE due to improved shielding and material selection.

We fit the ROI using a simultaneous UEML fit over all channels and datasets. We used the detector response function fit on the calibration data, scaled down to the ROI [11], to fit both the ${ }^{60} \mathrm{Co}$ peak at $2507 \mathrm{keV}$ and any potential $0 v \beta \beta$ peak. These are fit on top of a flat background. The resulting fit is shown in Fig. 3. We see no excess of events in the $0 v \beta \beta$ ROI, and out best fit rate is $\Gamma_{0 v}=(0.01 \pm 0.12$ (stat.) \pm 0.01 (syst.) $) \times 10^{-24} \mathrm{yr}^{-1}$. Which we combine with a uniform physical prior to form a lower Bayesian limit on the $0 v \beta \beta$ half-life of $T_{1 / 2}^{0 v}>2.7 \times 10^{24} \mathrm{yr}$ at $90 \%$ C.L. (including systematics [11]). This is consistent with our median expected $90 \%$ C.L. sensitivity of $2.9 \times 10^{24} \mathrm{yr}$.

We combine this result with the results of the Cuoricino experiment, to form a combined limit on the half-life of $T_{1 / 2}^{0 v}>4.0 \times 10^{24} \mathrm{yr}$ at $90 \%$ C.L. We use the most recent NME and phase space calculations $[18,19,20,21,22,23,24]$ to convert this limit onto an upper limit on the effective Majorana mass, $m_{\beta \beta}<270-760 \mathrm{meV}$ [10] (see Fig. 4).

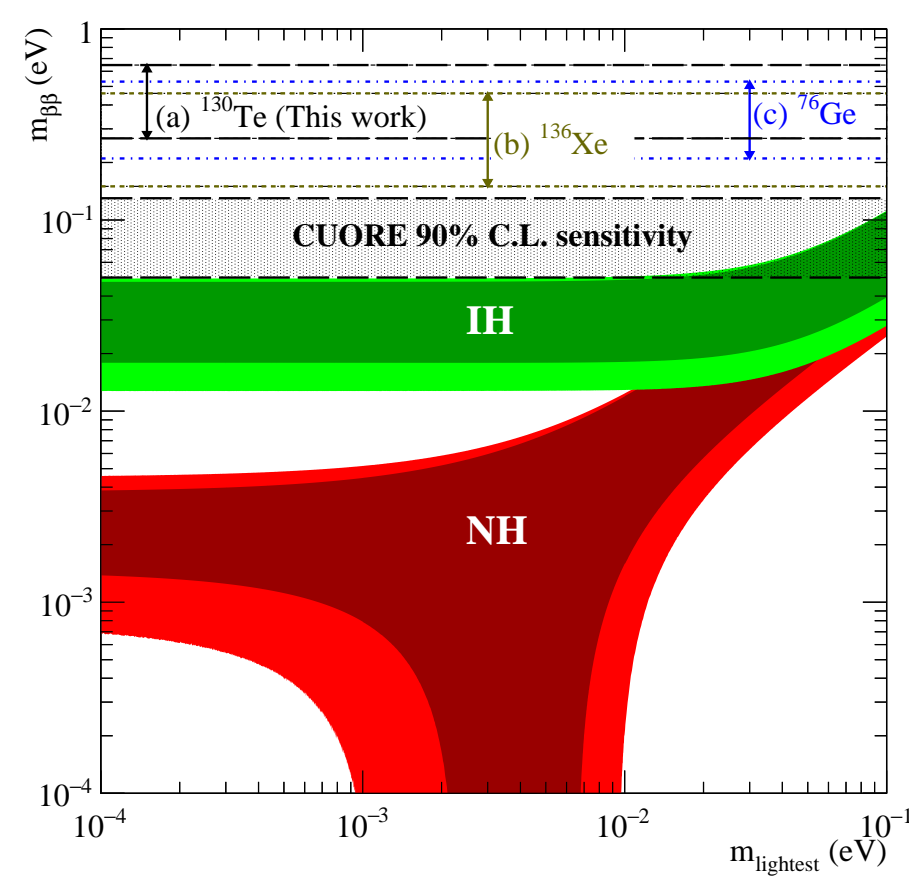

Figure 4: The allowed regions for $m_{\beta \beta}$ for the normal hierarchy (NH) and inverted hierarchy (IH). $90 \%$ confidence upper limits on the effective Majorana mass, $m_{\beta \beta}$, from experiments with ${ }^{76} \mathrm{Ge}[7],{ }^{136} \mathrm{Xe}[8,9]$ and ${ }^{130} \mathrm{Te}[10]$. The width of the upper limit is dominated by the uncertainty in the NME. The shaded area is the expected CUORE $90 \%$ sensitivity after 5 years of live time. 


\section{Discussion and Outlook}

\subsection{CUORE Outlook}

In CUORE-0, we see a factor of $\sim 6.8$ reduction in the rate of degraded $\alpha$ events. This reduction in background demonstrates the success of the background reduction techniques developed for CUORE. We expect the background rate from degraded $\alpha$ 's to be decreased further in CUORE due to the smaller amount of copper surface area per crystal. This gives confidence that the CUORE background goal of 0.01 counts/(keV. $\mathrm{kg} \cdot \mathrm{yr})$ in the ROI is within reach. With this background rate, and an energy resolution of $5 \mathrm{keV}$, CUORE will be sensitive to a ${ }^{130} \mathrm{Te} 0 v \beta \beta$ decay half-life of $9.5 \times 10^{25} \mathrm{yr}$ at the $90 \%$ C.L. within 5 years of live time. This corresponds to a $90 \%$ C.L. sensitivity range in $m_{\beta \beta}$ of $50-130 \mathrm{meV}$.

\subsection{Current Status of CUORE}

The construction of the CUORE detector was completed in summer of 2014. Since then, the detector has been stored in the CUORE clean room, underground, and under constant $N_{2}$ flux to minimize exposure to radon. In March of 2016, after the successful completion of the final commissioning run, the cryostat commissioning was declared completed. At the time of writing, the detector installation into the cryostat is on-going (see Fig. 5) and should be completed before the Fall of 2016. We hope to begin data taking before the end of 2016.

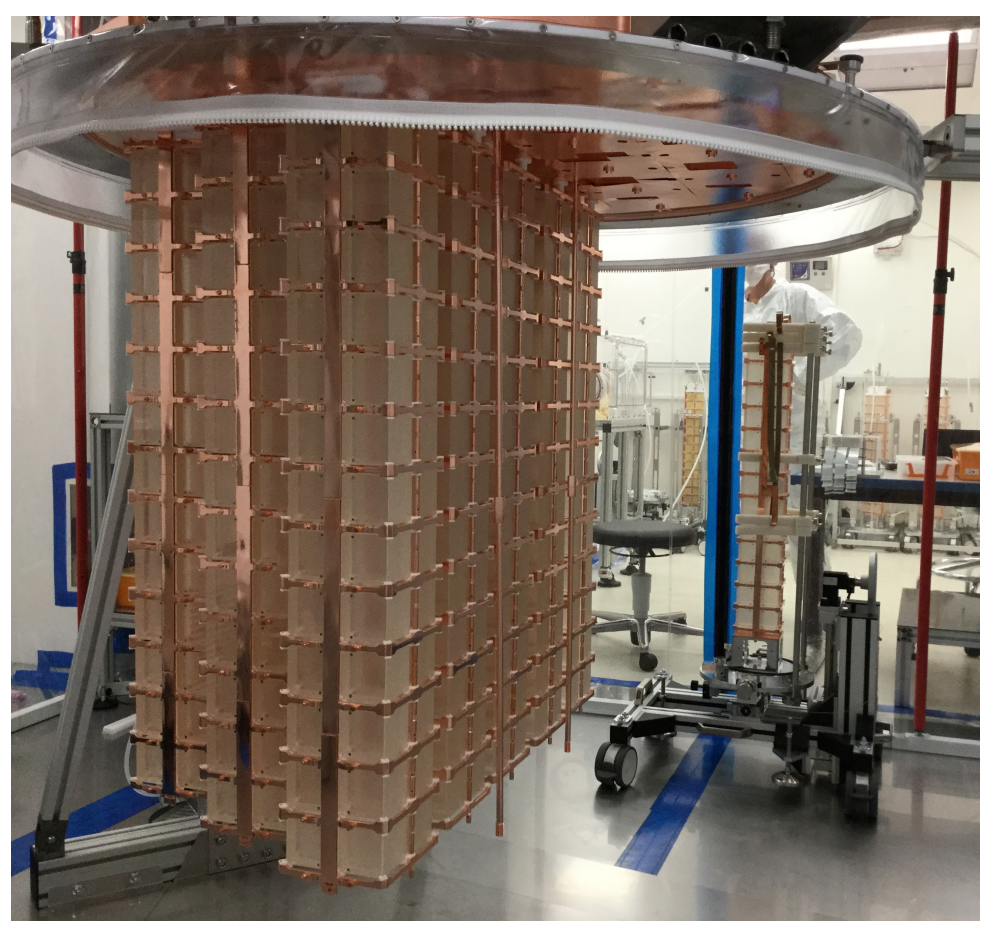

Figure 5: The first 12 towers of the CUORE detector installed in the CUORE cryostat. Photo taken right before attaching the protective shielding bag for the evening. 


\section{References}

[1] S. Fukuda et al. Phys. Lett. B, 539:179-187, 2002.

[2] Q. R. Ahmad et al. Phys. Rev. Lett., 87:071301, Jul 2001.

[3] Q. R. Ahmad et al. Phys. Rev. Lett., 89:011301, 2002.

[4] K. Eguchi et al. Phys. Rev. Lett., 90:021802, 2003.

[5] A. Gando et al. Phys. Rev. D, 83:052002, 2011.

[6] K. A. Olive et al. (Particle Data Group), Chin. Phys C, 38, 0900012014.

[7] M. Agostini et al. Phys. Rev. Lett., 111(12):122503, 2013.

[8] A. Gando et al. Phys. Rev. Lett., 110(6):062502, 2013.

[9] J.B. Albert et al. Nature, 510:229, 2014.

[10] K. Alfonso et al. Phys. Rev. Lett., 115:102502, 2015.

[11] C. Alduino et al. Phys. Rev. C, 93(4):045503, 2016.

[12] E. Andreotti et al. Astropart. Phys, 34:822, 2011.

[13] D. R. Artusa et al. Eur. Phys. J. C, 74:2956, 2014.

[14] C. Arnaboldi et al. J. Crys. Growth, 312:2999, 2010.

[15] F. Alessandria et al. Astropart. Phys., 35:839-849, 2012.

[16] F. Alessandria et al. Astropart. Phys, 45:13, 2013.

[17] C. Alduino et al. JINST, 11(07):P07009, 2016.

[18] J. Kotila and F. Iachello. Phys. Rev. C, 85:034316, 2012.

[19] J. Menendez et al. Nucl. Phys. A, 818:139, 2009.

[20] F. Ŝimkovic et al. Phys. Rev. C, 87:045501, 2013.

[21] J. Hyvrinen and J. Suhonen. Phys. Rev. C, 91:024613, 2015.

[22] J. Barea, J. Kotila, and F. Iachello. Phys. Rev. C, 91:034304, 2015.

[23] T. R. Rodriguez and G. Martinez-Pinedo. Phys. Rev. Lett, 105:252503, 2010.

[24] A. Neacsu and M. Horoi. Phys. Rev. C, 91:024309, 2015. 